\title{
Feasibility of subcutaneously implanted magnetic microarrays for site specific drug and gene targeting
}

\author{
A. Krafčík, P. Babinec and M. Babincová" \\ Department of Nuclear Physics and Biophysics, Comenius University, Mlynská dolina F1, 84248 Bratislava, Slovakia.
}

Received 30 December 2009; Revised 25 February 2010, Accepted 29 March 2010

\begin{abstract}
The magnetic nanoparticles play a crucial role as a drug carriers in the human body. The wedge like magnetic arrays creating a strongly non-homogeneous magnetic field are considered as a useful way to focus magnetic nanoparticles functionalized with various drugs or genes to desired sites. The goal of this study is to develop a numerical model of drug targeting using subcutaneously implanted magnetic microarrays. The Finite Element Method is applied to solve partial differential equations describing electromagnetic field (Maxwell equations) and motion of these particles in a given magnetic field is obtained solving set of ordinary differential equations expressed by Newton law of motion. The results are encouraging showing the potential to target drug to the tumour cell locally, without unwanted side effects.
\end{abstract}

Keywords: Electromagnetics, Maxwell equations, simulation, finite element method, drug targeting.

\section{Introduction}

Nanomedicine is the application of nanotechnology to medicine and is based on mutually overlapping and progressively more powerful molecular technologies [1]. Nanomedical developments range from nanoparticles for molecular diagnostics, imaging and therapy to integrated medical nanosystems, which may perform complex repair actions at the cellular level inside the body in the future. Magnetic drug targeting (MDT) is one of the new regional drug therapies presently investigated for cancer treatment [2-9]. The striking advantage of MDT is the reduction of side effects of chemotherapeutic agents for the patient due to applying the agents mainly to the desired target volume at the tumour. MDT is based on anticancer agents bound to ferromagnetic magnetite nanoparticles (diameter $15-400 \mathrm{~nm}$ ) with biocompatible starch coating suspended in a fluid [10-14]. The suspension is injected intra-arterially near the target volume. A magnet, positioned close to the tumour, drags the particles from the artery to the vasculature of the tumour and finally concentrates them in the tissue to be treated. The magnetic field has to be applied for a certain time, until the bond between the drug and the magnetic particle decays and the drug has penetrated the tumour-cells.

The goal of this study is to use Matlab communicating with FEMM software as an ActiveX client for comprehensive numerical analysis of implant assisted-magnetic drug targeting using the Finite Element Method (FEM) to solve partial differential equations describing electromagnetic field by Maxwell equations coupled to Newton law of motion given as a set of ordinary differential equations.

\footnotetext{
*E-mail address: babincova@fmph.uniba.sk
}

ISSN: 1791-2377 (C) 2010 Kavala Institute of Technology. All rights reserved.

\section{Theoretical model}

Forces acting on moving magnetic particle in magnetic field localized in viscous medium are of different origins. The most important are magnetic forces due to all magnetic sources and Stokes' viscous drag force. Several other interactions, like thermal kinetics (Brownian motion) or particle fluid interactions and interparticle effects, e.g. magnetic dipole interactions, electric double layer interactions, and van der Waals force, but together with inertia, buoyancy and gravity, are their contributions to the overall force acting on particle negligible. Therefore we can consider in this paper only the magnetic and viscous drag forces. Trajectories of motion of magnetic particles in magnetic field and viscous fluid can be calculated using Newton's law:

$$
m_{p} \frac{d \mathbf{v}_{p}}{d t}=\mathbf{F}_{m}+\mathbf{F}_{s}
$$

where $\mathrm{mp}$ and vp are the mass and velocity of the particle, and Fm and Fs are the magnetic and Stokes' drag forces, respectively

\subsection{Magnetic force}

For the determination of magnetic force acting on magnetic particle we use method of "effective" dipole moment in which a spherical magnetic particle is replaced by "equivalent" point dipole moment $\mathbf{m}_{p, e f f}$ localized at the centre of particle. Its value 
depends on magnitude of intensity of applied field $\mathbf{H}_{a}$, rising from model based on self-demagnetization and magnetic saturation of magnetite particles

$$
\mathbf{F}_{m}=\mu\left(\mathbf{m}_{p, e f f} \cdot \nabla\right) \mathbf{H}_{a}
$$

where $\mu$ is permeability of fluid environment. Magnetic force is therefore function of external magnetic field gradient and the magnetization of the particle. Before saturation, particles are linearly magnetized with their magnetic moment magnitude increasing in the direction of the external field. Beyond the saturation point, magnetic moment magnitude tends to a constant value. In this model the effective dipole moment can be expressed as

$$
\mathbf{m}_{p, e f f}=V_{p} f\left(H_{a}\right) \mathbf{H}_{a}
$$

where we consider magnetic particle with radius $R_{p}$ and volume $V_{p}=4 / 3 \pi R_{p}^{3}$ and a function

$$
f\left(H_{a}\right)=\left\{\begin{array}{ll}
\frac{3\left(\chi_{p}-\chi_{f}\right)}{\left(\chi_{p}-\chi_{f}\right)+3}, & H_{a}<\left(\frac{\left(\chi_{p}-\chi_{f}\right)+3}{3 \chi_{p}}\right) M_{s p} \\
M_{s p} / H_{a}, & H_{a} \geq\left(\frac{\left(\chi_{p}-\chi_{f}\right)+3}{3 \chi_{p}}\right) M_{s p}
\end{array}\left(\left|\chi_{f}\right|<<1\right)\right.
$$

where $\chi_{p}$ and $\chi_{f}$ are the magnetic susceptibilities of the particle and ambient fluid, respectively, $M_{s p}$ is the saturation magnetization of the particle, and $H_{\alpha}=\left|\mathbf{H}_{\alpha}\right|$.

If we assume nonmagnetic fluid $\left(\chi_{f}=0\right)$ and magnetic particles with high susceptibility $\left(\chi_{p}>>1\right)$, what is in the case of water as fluid environment and magnetite $\left(\mathrm{Fe}_{3} \mathrm{O}_{4}\right)$ as particles accomplished, then $f\left(H_{a}\right)$ or better $f(B)$ is

$$
f(B)=\left\{\begin{array}{cc}
3, & B / \mu<M_{s p} / 3 \\
M_{s p} \mu / B, & B / \mu \geq M_{s p} / 3
\end{array}\right.
$$

where $B$ is magnetic flux density of external field and is valid: $B / \mu=H_{\alpha}$.

\subsection{Magnetic field of permanent magnets}

Sources of magnetic field, like permanent magnets, and surrounding material could be modelled by finite elements method. The simplest way is to consider magnetostatic problem, i.e. the timeindependent fields. In this approach, the field intensity $(\mathbf{H})$ and flux density (B), with relation $\mathbf{B}=\mu \mathbf{H}$, limit Maxwell's equations on two of them

$$
\begin{aligned}
& \nabla \times \mathbf{H}=\mathbf{J} \\
& \nabla \cdot \mathbf{B}=0
\end{aligned}
$$

where $\mathbf{J}$ is current density. According mentioned above and via a magnetic vector potential (A) approach, i.e.

$$
\mathbf{B} \equiv \nabla \times \mathbf{A}
$$

equation (6), in the case of nonlinear material, when permeability is a function of $\mathrm{B}$, becomes

$$
\nabla \times\left(\frac{1}{\mu(\mathbf{B})} \nabla \times \mathbf{A}\right)=\mathbf{J}
$$

For a linear isotropic material $(\mu=$ const. $)$ will previous expression reduce in Coulomb gauge $(\nabla \cdot \mathbf{A}=0)$ to

$$
-\nabla \cdot\left(\frac{1}{\mu} \nabla\right) \mathbf{A}=\mathbf{J}
$$

and, if we consider problems as planar ones, $B_{z}=0$, the last equation leads to scalar elliptic partial differential equation

$-\nabla \cdot\left(\frac{1}{\mu} \nabla A\right)=J$

that numerical solution using FEM [15] is the basis of David Meeker FEMM [16] program used in this study for magnetic flux density determination. An excellent review of the FEMM capabilities has been recently published in this journal by Baltzis [17].

\section{Stokes' drag force}

In our model we consider in addition to magnetic force also fluidic force acting on moving particle in fluid medium. Its magnitude is determined by Stokes' law for the drag on a sphere with radius $R_{p}$ in uniform flow,

$$
\mathbf{F}_{s}=-6 \pi \eta R_{p}\left(\mathbf{v}_{p}-\mathbf{v}_{f}\right)
$$

where $\eta$ and $\mathbf{v}_{f}$ are the viscosity and the velocity of the flu$\mathrm{id}$, respectively, and $\mathbf{v}_{p}$ is the velocity of the particle. Spherical magnetic micro- and nanoparticles are moving in capillary which diameter is much greater than the size of particle. Velocity of flowing fluid in capillary with radius $R_{c}$ is determined from NavierStokes equation of Poiseuille-flow problem of Newtonian fluid with no-slip boundary condition [18]. In terms of average speed $\bar{v}_{f}$ of flowing fluid we can write

$v_{f}(r)=2 \bar{v}_{f}\left(1-\frac{r^{2}}{R_{c}^{2}}\right)$

where $v_{f}(r)$ is velocity of fluid in axial direction of capillary in distance $r$ from its axis. If we consider planar problem, holds in Cartesian coordinates system, with $x$ coordinate axis identical with capillary axis, that: $y=r$, and

$$
\begin{aligned}
& v_{x}=v_{f}(y), \\
& v_{y}=v_{z}=0,
\end{aligned}
$$

i.e. infinite capillary is replaced by infinite parallel-plate channel.

We have also considered configuration of magnetic field sources with non-moving fluid medium. In this case is the fluid ambient quiescent, i.e. $v_{f}(y)=0 \mathrm{~m} \cdot \mathrm{s}^{-1}$. 


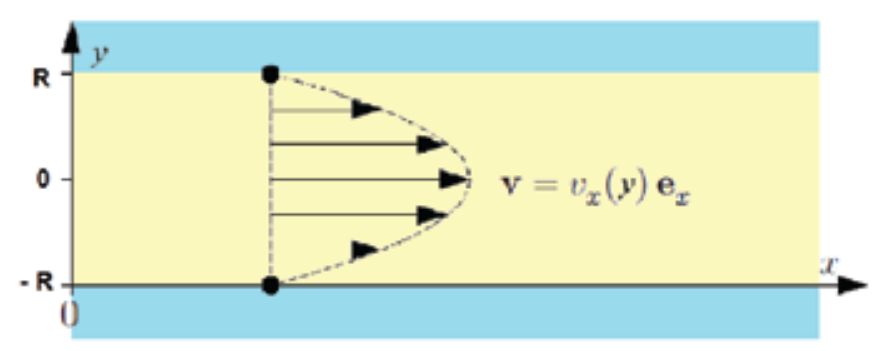

Figure 1. Structure of Poiseuille flow in the $x y$-plane of an infinite parallelplate channel of the height $2 R[18]$.

\subsection{Equations of motion}

In the next step computed magnetic flux density of studied arrangements of permanent magnets by FEM analysis were extracted from FEMM program and used for calculation of trajectories. For these purposes we have used OctaveFEMM (available from D. Mekker FEMM web page [16]) which is a Matlab toolbox that allows for the operation of FEMM via a set of Matlab functions. The toolbox works with Octave, a free Matlab clone. Since Octave does not support ActiveX, the toolbox use an alternative interprocess communication scheme based on temporary files for passing messages between Octave and FEMM. This toolbox can also run in Matlab using Matlab's built-in support of ActiveX. The use and functionality of the toolbox is essentially identical under either Matlab or Octave. The syntax of the OctaveFEMM toolbox closely mirrors that of FEMM's existing Lua [19] scripting language interface associated with FEMM.

Movement of magnetic particles in the plane in magnetic field with flux density $B$ in flowing Newtonian fluid with viscosity $\eta$ is finally described by system of ordinary differential equations

$$
\begin{aligned}
\frac{d x}{d t} & =v_{p, x} \\
\frac{d y}{d t}= & v_{p, y} \\
\frac{d v_{p, x}}{d t}= & \frac{1}{m_{p}}\left\{\frac { V _ { p } } { \mu _ { f } } f ( B ( x , y ) ) \left[+B_{x}(x, y) \frac{\partial B_{x}(x, y)}{\partial x}+\right.\right. \\
+ & \left.\left.B_{y}(x, y) \frac{\partial B_{x}(x, y)}{\partial y}\right]-6 \pi \eta R_{p}\left[v_{p, x}-2 \bar{v}_{f}\left(1-\frac{y^{2}}{R_{c}^{2}}\right)\right]\right\} \\
\frac{d v_{p, y}}{d t}= & \frac{1}{m_{p}}\left\{\frac { V _ { p } } { \mu _ { f } } f ( B ( x , y ) ) \left[+B_{x}(x, y) \frac{\partial B_{y}(x, y)}{\partial x}+\right.\right. \\
& \left.\left.+B_{y}(x, y) \frac{\partial B_{y}(x, y)}{\partial y}\right]-6 \pi \eta R_{p} v_{p, y}\right\}
\end{aligned}
$$

where $m_{p}$ and $V_{p}$ are the mass and the volume of particle, respectively. Gradient of flux density components was calculated numerically in each point of trajectory from the definition by replacing limit of space element by element of dimension ten times smaller than maximal size of mesh elements of the FEM model.

\section{Results and discussion}

We have analyzed magnetite $\left(\mathrm{Fe}_{3} \mathrm{O}_{4}\right)$ particles, with density $\rho_{p}$ $=5000 \mathrm{~kg} \cdot \mathrm{m}^{-3}$ and a saturation magnetization $M_{s p}=4.78 \times 10^{5}$ A. $\mathrm{m}^{-1}$, in the magnetic field of a given arrangements of permanent neodymium magnets, in moving fluid medium, described by Poiseuille flow in infinite parallel-plate channel as well as for non-moving fluid ( $\left.\bar{v}_{f}=0 \mathrm{~m} \cdot \mathrm{s}^{-1}\right)$. Theoretical model described in previous section have been used with ode23s numerical solver of software Matlab 7.0 (The MathWorks, 2004).

Computed trajectories of magnetite particles with radius 50 $\mathrm{nm}$ and $10 \mu \mathrm{m}$ moving in flowing water with average velocity 1 $\mathrm{mm} . \mathrm{s}^{-1}$ in the field of small magnets with millimetre dimensions are shown in Fig. 2. As can be seen particles are attracted to the margin of capillary in the location of applied field depending on the initial position, initial velocity, and size of particle. Potential applications of such a small magnets for targeting of magnetic particles is especially important when using particles with sizes in micrometers, having capture radius of magnetic field in flowing water small, due to the fact that particles are dragged by viscous
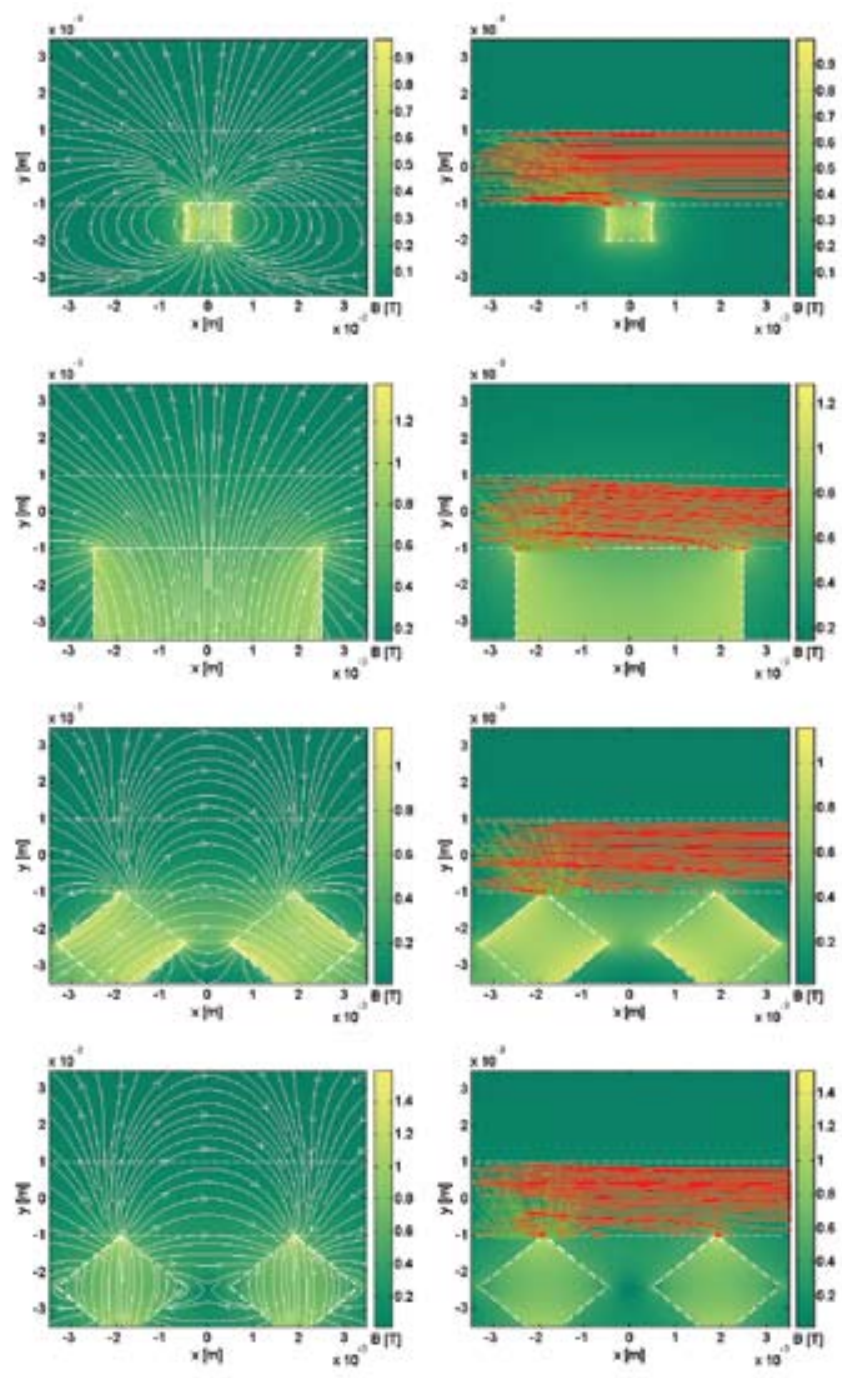

Figure 2. Magnetic flux densities of small magnets and trajectories of microparticles (green; radius $10 \mu \mathrm{m}$ ) as well as nanoparticles (red; radius $50 \mathrm{~nm}$ ) in the flowing water described by Poiseuille flow in infinite parallel-plate channel, as a model of flow capillary, with average speed $1 \mathrm{~mm} \cdot \mathrm{s}^{-1}$. 
forces of the moving fluid. But it is necessary to remark, that for instance in real blood supply of target tissue or tumour the capillaries are of small diameter, therefore tumour embolization should also be taken into account. On the other hand when we consider blood vessels with the same diameter as in our calculations the average speed of flowing blood is in orders of a few cm. $\mathrm{s}^{-1}$.

Further we have studied FEM model of high gradient magnetic field in the vicinity of ferromagnetic dots with radius $5 \mu \mathrm{m}$ placed in a homogeneous external magnetic field (HMP). As a ferromagnetic material we used supermalloy in HMP with a moderate flux density $0.46 \mathrm{~T}$ (Fig. 3).
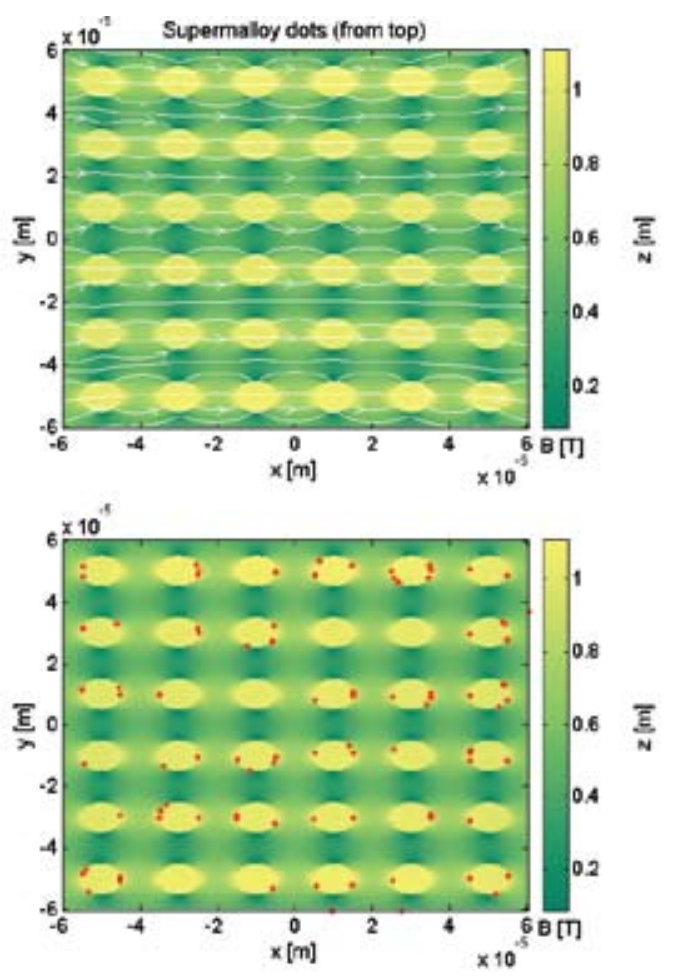

Figure 3. Flux density with a snapshot of trajectories of magnetite nanoparticles (radius $50 \mathrm{~nm}$ ) in the vicinity of supermalloy dots in homogeneous magnetic field with magnitude $0.46 \mathrm{~T}$ in the water. View from the top and profile.
Mean capture time needed for attraction of magnetite nanoparticles (radius $50 \mathrm{~nm}$ ) by such a dots is approximately $15 \mathrm{~ms}$ and effective capture radius is $20 \mu \mathrm{m}$, thus the distance is comparable to cell sizes, which means that such arrangement is feasible for in vitro targeting of effective compounds (e.g. DNA or siRNA [20]) carried by magnetic nanoparticles into cells cultured on the surface with ferromagnetic dots. Such dots could be also used in vivo as subcutaneously implanted micro targeting system on surface of vessels or tumours for effective drug capturing.

\section{Conclusions}

The ultimate goal of magnetic drug targeting is to achieve a high concentration of drug in the target region. In this study, which from the computational point of view can be considered as a continuation of Baltzis review [17], we have shown that using FEMM program package several important questions of this expanding field of biomedical research can be addressed theoretically before complicated and expensive experiments on living animals and we hope that these findings will provide a useful basis for future magnetic implants design for effective magnetic targeting of therapeutics in vivo.

\section{Acknowledgments}

This work was supported by VEGA grant 1/0082/08 and Magselectofection project of 6. FP of EU under the contract No.: LSHBCT-2006-019038.

\section{References}

1. K. K. Jain, The Handbook of Nanomedicine, Humana Press, Totowa, NJ, USA, (2008).

2. M. Babincová, Microwave induced leakage of magnetoliposomes. Possible clinical implications, Bioelectrochemistry and Bioenergetics, 32, pp. 187-190, (1993).

3. M. Babincová and P. Babinec, Controlled drug delivery using magnetoliposomes, Cellular and Molecular Biology Letters, 2, pp. 3-9, (1997).

4. M. Babincová, V. Altanerová, M. Lambert, Č. Altaner, M. Šramka, E. Machová and P. Babinec, Site specific in vivo targeting of magnetoliposomes in external magnetic field, Zeitschrift für Naturforschung, 55c, pp. 278$284,(2000)$

5. M. Babincová, D. Leszczynska, P. Sourivong, P. Babinec and J. Leszczynski, Principles of magnetodynamic chemotherapy, Medical Hypotheses, 62 , pp. 375-377, (2004).
6. P. Babinec, M. Babincová, P. Sourivong and D. Leszczynska, Efficient treatment of pigmented B16 melanoma using photosensitized long-circulating magnetofullerenosomes, Journal of Magnetism and Magnetic Materials, 293, pp. 394-397, (2005).

7. M. Babincova, V. Altanerova, C. Altaner, C. Bergemann and P. Babinec, In vitro analysis of cisplatin functionalized magnetic nanoparticles in combined cancer chemotherapy and electromagnetic hyperthermia, IEEE Transactions on Nanobioscience, 7, pp. 15-19, (2008).

8. M. Babincová, P. Babinec and C. Bergemann, High-gradient magnetic capture of ferrofluids: Implications for drug targeting and tumor embolization, Zeitschrift fur Naturforschung - Section C Journal of Biosciences vol. 56, pp. 909-911, (2001).

9. A. Krafčík, M. Babincová and P. Babinec, Theoretical analysis of magnetic particle trajectory in high-current pulsed quadrupole: implications 
for magnetic cell separation, drug targeting, and gene therapy, Optoelectronics and Advanced Materials - Rapid Communications, 3, pp. 226-233, (2009).

10. A. Munir, J. Wang, H. S. Zhou, Dynamics of capturing process of multiple magnetic nanoparticles in a flow through microfluidic bioseparation system, IET Nanobiotechnology, 3, pp. 55-64, (2009).

11. S. Shaw, P. V. S. N. Murthy, S. C. Pradhan, Effect of non-Newtonian characteristics of blood on magnetic targeting in the impermeable micro-vessel, Journal of Magnetism and Magnetic Materials, 322, pp. 1037-1043, (2010).

12. B. Gleich, T. Weyh, B. Wolf, Magnetic drug targeting: An analytical model for the influence of blood properties on particle trajectories, Applied Rheology, 18, pp. 52023-1-52023-7, (2008).

13. E. J. Furlani, E. P. Furlani, A model for predicting magnetic targeting of multifunctional particles in the microvasculature, Journal of Magnetism and Magnetic Materials, 312, pp. 187-193, (2007).

14. T. Knopp, S. Biederer, T. Sattel, J. Weizenecker, B. Gleich, J. Borgert, T.
M. Buzug, Trajectory analysis for magnetic particle imaging, Physics in Medicine and Biology, 54, pp. 385-397, (2009).

15. J. L. Volakis, A. Chatterjee and L. C. Kempel, Finite Element Methods for Electromagnetics, IEEE Press, USA, (1998).

16. D. Meeker, Finite Elements Methods Magnetics, V 4.2.; http://femm. foster-miller.net.

17. K. B. Baltzis, The FEMM Package: A Simple, Fast, and Accurate Open Source Electromagnetic Tool in Science and Engineering, Journal of Engineering Science and Technology Review, 1, pp. 83-89, (2008).

18. H. Bruus, Theoretical Microfluidics, Oxford University Press, Oxford, UK, (2008).

19. R. Ierusalimschy, Programming in Lua, 2nd ed., Biblioteca do Departamento de Informática, (2006); http://www.lua.org.

20. U. Schillinger, T. Brill, C. Rudolph, S. Huth, S. Gersting, F. Krötz, J. Hirschberger, C. Bergemann and C. Plank, Advances in magnetofection - magnetically guided nucleic acid delivery, Journal of Magnetism and Magnetic Materials, 293, pp. 501-508, (2005). 